\title{
A New Multivariable Grey Convolution Model Based on Simpson's Rule and Its Applications
}

\author{
Song Ding $\mathbb{D}^{1,2}$ and Ruojin $\mathrm{Li} \mathbb{D}^{1}$ \\ ${ }^{1}$ School of Economics, Zhejiang University of Finance \& Economics, Hangzhou 211106, China \\ ${ }^{2}$ Center for Regional Economy \& Integrated Development, Zhejiang University of Finance \& Economics, \\ Hangzhou 310018, China
}

Correspondence should be addressed to Ruojin Li; lrjxueshu@163.com

Received 24 August 2019; Accepted 4 February 2020; Published 27 February 2020

Academic Editor: Mojtaba Ahmadieh Khanesar

Copyright (C) 2020 Song Ding and Ruojin Li. This is an open access article distributed under the Creative Commons Attribution License, which permits unrestricted use, distribution, and reproduction in any medium, provided the original work is properly cited.

\begin{abstract}
Accurate estimations can provide a solid basis for decision-making and policy-making that have experienced some kind of complication and uncertainty. Accordingly, a multivariable grey convolution model (GMC $(1, n)$ ) having correct solutions is put forward to deal with such complicated and uncertain issues, instead of the incorrect multivariable grey model $(\mathrm{GM}(1, n))$. However, the conventional approach to computing background values of the GMC $(1, n)$ model is inaccurate, and this model's forecasting accuracy cannot be expected. Thereby, the drawback analysis of the GMC $(1, n)$ model is conducted with mathematical reasoning, which can explain why this model is inaccurate in some applications. In order to eliminate the drawbacks, a new optimized GMC $(1, n)$, shorted for OGMC $(1, n)$, is proposed, whose background values are calculated based on Simpson' rule that is able to efficiently approximate the integration of a function. Furthermore, its extended version that uses the Gaussian rule to discretize the convolution integral, abbreviated as $\operatorname{OGMC}_{\mathrm{G}}(1, n)$, is proposed to further enhance the model's forecasting ability. In general, these two optimized models have such advantages as simplified structure, consistent forecasting performance, and satisfactory efficiency. Three empirical studies are carried out for verifying the above advantages of the optimized model, compared with the conventional GMC $(1, n), \operatorname{GMC}_{\mathrm{G}}(1, n), \mathrm{GM}(1, n)$, and DGM $(1, n)$ models. Results show that the new background values can effectively be calculated based on Simpson's rule, and the optimized models significantly outperform other competing models in most cases.
\end{abstract}

\section{Introduction}

Grey system theory has gained extensive attentions from worldwide researchers and has been successfully used in many fields with favorable outcomes since it was designed by Deng in 1982 [1-3]. This theory is capable of addressing issues characterized by uncertainty, insufficient information, and limited data points, thereby providing strong technical support for uncertain analysis $[4,5]$. As the most important part of this theory, grey prediction approach and its extended versions have captured enormous attention owing to their capability to provide accurate forecasts, especially under the situation that facing sparse data and poor information $[6,7]$. In contrast to the intelligent techniques and traditional statistical models, which require a large amount of data for model calibration, grey models only need limited data points to estimate the system behavior.

Among the grey-model groups, the $\operatorname{GM}(1, n)$ model, of which 1 means one order of differential function and $n$ stands for the number of total variables, gains its reputation and operates as a typical forecasting technique due to its capability to deal with the system prediction issues that are influenced by a range of relevant factors. Unfortunately, as the solutions to the whitening function for $\operatorname{GM}(1, n)$ are rough and incorrect [8], it is prone to produce large errors in applications. Thereby, since its introduction, many researchers carried out studies for further improve its prediction performance in terms of the background values [9], discrete variants [10], model structures [11], fractional model [12], and time-delayed versions [13]. Out of these, a 
novel grey prediction model with convolution integral, namely, $\operatorname{GMC}(1, n)$, was designed by Tien [14] with a view to avoiding the incorrect solutions of the $\operatorname{GM}(1, n)$ model. As Tien presented, a convolution integral exists in the analytic solution to the whitening function of $\operatorname{GM}(1, n)$, and the Trapezoid formula is used to discretize the convolution integral. Many cases have proved that the $\operatorname{GMC}(1, n)$ model outperforms the typical $\operatorname{GM}(1, n)$. Moreover, for further adapting to the sequences having various characteristics, a range of variants derived from the $\operatorname{GMC}(1, n)$ model is put forward, such as $\operatorname{DGDMC}(1, n)$ [15-19] $\operatorname{IGDMC}(1, n)$, $\operatorname{FGMC}(1, n), \operatorname{NGMC}(1, n), \operatorname{GMCN}(1, n)$, and kernel-based nonlinear $\operatorname{GMC}(1, n)[20]$.

In addition to these extended versions, higher accuracy grey approaches are typically obtained by reconstructing the background values, which are of great importance to the accurate parameter estimations. Essentially, the background values are used to smooth data to reduce randomness further [9] and customarily are represented by the average values of adjacent neighbor 1-AGO sequences, namely, $Z_{i}^{(1)}(t)=$ $0.5\left[X_{i}^{(1)}(t)+X_{i}^{(1)}(t-1)\right], i=1,2, \ldots$. Detailed discussions on the reasonability and practicability have been carried out from diverse perspectives $[1,21,22]$. However, the rough estimations of background value are prone to give rise to the inconsistency of the grey differential and continuous equations, which results in the inaccurate forecasting performance of the target model. In order to improve the model's performance, Wang and Hao [23] incorporate the dynamic coefficients $\lambda_{i} \in[0,1](i=1,2, \ldots, n)$ into the structure of background values in $\operatorname{GMC}(1, n)$, namely, the background values for the system behavior sequence $Z_{1}^{(1)}(r p+t)=\lambda_{1} X_{1}^{(1)}(r p+t)+\left(1-\lambda_{1}\right) X_{1}^{(1)}(r p+t-1)$ and the background values for the relevant sequences $Z_{i}^{(1)}(t)=\lambda_{i} X_{i}^{(1)}(t)+\left(1-\lambda_{i}\right) X_{i}^{(1)}(t-1), i=2,3, \ldots$ Subsequently, for the purpose of further enhancing precision, various heuristic intelligent techniques are employed to determine the background value coefficient, including Particle Swarm Optimization (PSO) [24-26], Genetic Algorithm (GA) [27, 28], Ant Lion Optimizer (ALO) [29], and Ant Colony Algorithm (ACA) [30].

Though the previous improvements in background values with the support of those heuristic intelligent techniques lead to the improvements of forecasting ability, they also result in the low-level, simply-repeated, and complicated computations of grey models. Meanwhile, it is troublesome and difficult for beginners or inexperienced users to choose a suitable heuristic intelligent algorithm for solving actual forecasting problems. Therefore, proposing an accurate forecasting grey model that has a simple structure and easy-understanding computations with a focus on the background values is a very valuable work. General consensus gotten from the literature was that accurate background values are regarded as a crucial requirement to ensure the reliability and practicability of the $\operatorname{GMC}(1, n)$ model $[23,24]$, and the forecasting precision is heavily dependent on their correct computations. Supporting this argument, one of the major contributions in this paper stems from the mathematical derivation of the actual background values, finding its differences from the estimated one in the conventional GMC $(1, n)$ model. Another equally important merit of this paper is to design an optimized $\operatorname{OGMC}(1, n)$, whose background values are reconstructed and calculated based on Simpson's rule. Moreover, the new model's extended version is also derived by using the Gaussian rule, instead of the Trapezoidal rule, to calculate the whitening function with convolution integral, namely, $\mathrm{OGMC}_{\mathrm{G}}(1, n)$. Subsequently, three cases of diverse subjects, including tensile strength of a material, China's gross industrial output value, and electronic waste in Washington State, are utilized for verifying the efficacy and robustness of the proposed models. Empirical results, the optimized background values, based on Simpson's rule can act as a supplementary for the current estimation methods of grey models. Furthermore, the $\operatorname{OGMC}_{\mathrm{G}}(1, n)$ model having optimized background values performs best among all competitors. The detailed comparisons will be elaborated in Section 3.

This work has the following structure. The research methodology is presented in Section 2. In this section, the focus is on the previous procedures and shortcomings of the $\operatorname{GMC}(1, n)$ model. Subsequently, the optimized $\operatorname{OGMC}(1, n)$ and $\operatorname{OGMC}_{\mathrm{G}}(1, n)$ models are elaborated on. Section 3 is dedicated to three different cases for depicting the accuracy and reliability of the proposed models in comparison with a range of competitors. Conclusions and discussions are provided in the last Section 4.

The nomenclature utilized in this paper is presented below and detailed explanations related to the formulas employed in each technique are given in the following sections.

\section{Methodology}

2.1. Overview of the GMC $(1, n)$ Model. The GMC $(1, n)$ model has gains popularity in practical applications because of its accurate estimations and simple structures. Many researchers such as Tien [14-17], Ma et al. [31, 32], and Duman et al. [24], have elaborated the procedures for this model, which can currently be outlined below:

Step 1: collect the original data. Assume that $X_{1}^{(0)}=$ $\left[x_{1}^{(0)}(r p+1), x_{1}^{(0)}(r p+2), \ldots, x_{1}^{(0)}(r p+r)\right], r \geq 4$ is a data sequence of a system's characteristic variable, short for the system behavior sequence, and $X_{i}^{(0)}=\left[x_{i}^{(0)}(1), x_{i}^{(0)}(2), \ldots, x_{i}^{(0)}(r)\right], r \geq 4, i=2,3, \ldots, n$ are the data sequences of relevant factors, abbreviated as the relevant series. In the series, $r$ represents the number of entries for establishing the model, $r p$ stands for the delayed period, and $n$ is the total number of variables. Moreover, these two categories of series are nonnegative and equally spaced over time.

Step 2: generate the $X^{(1)}$ by using the first-order accumulated generating operation $(1-\mathrm{AGO})$. Then the accumulated sequences $X_{i}^{(1)}, i=1,2, \ldots, n$ of the original data $X_{i}^{(0)}(i=1,2, \ldots, n)$ can be produced by using 


$$
\begin{aligned}
x_{1}^{(1)}(r p+t) & =\sum_{j=1}^{r p+t} x_{1}^{(0)}(r p+j), \quad t=1,2, \ldots, r p+r \\
x_{i}^{(1)}(t) & =\sum_{j=1}^{t} x_{1}^{(0)}(j) .
\end{aligned}
$$

Accordingly, the $1-\mathrm{AGO}$ sequences of the system behavior can be obtained as

$X_{1}^{(1)}=\left[x_{1}^{(1)}(r p+1), x_{1}^{(1)}(r p+2), \ldots, x_{1}^{(1)}(r p+r)\right]$.

And the 1-AGO sequences of the influencing factors are

$X_{i}^{(1)}=\left[x_{i}^{(1)}(1), x_{i}^{(1)}(2), \ldots, x_{i}^{(1)}(r)\right], \quad i=2,3, \ldots, n$.

Step 3: build the $\operatorname{GMC}(1, n)$ model. Its first-order whitening equation is presented by

$\frac{\mathrm{d} X_{1}^{(1)}(r p+t)}{\mathrm{d} t}+\beta_{1} X_{1}^{(1)}(r p+t)=\sum_{i=2}^{n} \beta_{i} X_{i}^{(1)}(t)+u$,

where $\beta_{1}$ and $u$ represent the system's development coefficient and grey control parameter, respectively. Additionally, $\beta_{i} X_{i}^{(1)}(t)$ is known as the driving term, and $\beta_{i}, i=2,3, \ldots, n$ are the driving coefficients corresponding to the relevant factor sequences $X_{i}^{(0)}, i=2,3, \ldots, n$, respectively.

The grey derivative in equation (5) can be approximately estimated by

$$
\begin{aligned}
\frac{\mathrm{d} X_{1}^{(1)}(r p+t)}{\mathrm{d} t} & =\lim _{\Delta t \longrightarrow 0} \frac{X_{1}^{(1)}(r p+t)-X_{1}^{(1)}(r p+t-\Delta t)}{\Delta t} \\
& =X_{1}^{(1)}(r p+t)-X_{1}^{(1)}(r p+t-1) \\
& =X_{1}^{(0)}(r p+t) .
\end{aligned}
$$

In addition, the background values of $X_{1}^{(1)}(r p+t)$ and $X_{1}^{(1)}(r p+t)$ are usually taken as

$$
\begin{aligned}
Z_{1}^{(1)}(r p+t) & =0.5\left[X_{1}^{(1)}(r p+t)+X_{1}^{(1)}(r p+t-1)\right] \\
Z_{i}^{(1)}(t) & =0.5\left[X_{i}^{(1)}(t)+X_{i}^{(1)}(t-1)\right] .
\end{aligned}
$$

However, recent studies $[9,23,24]$ has pointed out that, the estimated values of the background value may be unequal to the actual values, explained in Section 2.2. Hence, based on the background values, the first-order grey differential equation can be formulated as

$X_{1}^{(0)}(r p+t)+\beta_{1} Z_{1}^{(1)}(r p+t)=\sum_{i=2}^{n} \beta_{i} Z_{i}^{(1)}(t)+u$.

Step 4: estimate the parameters by using the Ordinary Least Squares method (OLS). Substituting the values of $t$ into equation (9), we can obtain

$$
\begin{aligned}
& X_{1}^{(0)}(r p+2)+\beta_{1} Z_{1}^{(1)}(r p+2)=\sum_{i=2}^{n} \beta_{i} Z_{i}^{(1)}(2)+u \\
& X_{1}^{(0)}(r p+3)+\beta_{1} Z_{1}^{(1)}(r p+3)=\sum_{i=2}^{n} \beta_{i} Z_{i}^{(1)}(3)+u \\
& \vdots \\
& X_{1}^{(0)}(r p+r)+\beta_{1} Z_{1}^{(1)}(r p+r)=\sum_{i=2}^{n} \beta_{i} Z_{i}^{(1)}(r)+u .
\end{aligned}
$$

In this matrix form, $Y=B \widehat{\beta}$, where

$$
\begin{aligned}
Y & =\left[\begin{array}{c}
X_{1}^{(0)}(2) \\
X_{1}^{(0)}(3) \\
\vdots \\
X_{1}^{(0)}(r)
\end{array}\right], \\
B & =\left[\begin{array}{ccccc}
-Z_{1}^{(1)}(r p+2) & Z_{2}^{(1)}(2) & \cdots & Z_{n}^{(1)}(2) & 1 \\
-Z_{1}^{(1)}(r p+3) & Z_{2}^{(1)}(3) & \cdots & Z_{n}^{(1)}(3) & 1 \\
\vdots & \vdots & \ddots & \vdots & \vdots \\
-Z_{1}^{(1)}(r p+r) & Z_{2}^{(1)}(r) & \cdots & Z_{n}^{(1)}(r) & 1
\end{array}\right] .
\end{aligned}
$$

By solving the matrix $Y=B \widehat{\beta}$, the estimated values of the parameters can be calculated:

$$
\widehat{\beta}=\left[\widehat{\beta}_{1}, \widehat{\beta}_{2}, \ldots, \widehat{\beta}_{n}, \widehat{u}\right]=\left(B^{T} B\right)^{-1} B^{T} Y .
$$

Step 5: obtain the response function for projections. As introduced above, the whitening equation of the $\operatorname{GMC}(1, n)$ model is displayed in equation (5). By substituting the parameter values $\widehat{\beta}=\left[\widehat{\beta}_{1}, \widehat{\beta}_{2}, \ldots, \widehat{\beta}_{n}, \widehat{u}\right]$ into equation (5) and opting the $\widehat{X}_{1}^{(1)}(r p+1)=X_{1}^{(1)}(r p+1)$ as the initial condition, the time response function can be obtained by solving the whitening equation in equation (5), which will be presented as 
$\widehat{X}_{1}^{(1)}(r p+t)=X_{1}^{(01)}(r p+1) e^{-\beta_{1}(t-1)}+\int_{1}^{t} e^{-\beta_{1}(t-\tau)} f(\tau) \mathrm{d} \tau$,

where

$$
f(\tau)=\sum_{i=2}^{n} \beta_{i} X_{i}^{(1)}(\tau)+u
$$

As Tien [14] introduced previously, the Trapezoidal rule is originally used to discretize the convolution integral in equation $(13)$ in the traditional $\operatorname{GMC}(1, n)$ model, and subsequently, the response function for $1-$ AGO sequences can be determined by using the following equation:

$$
\begin{aligned}
\widehat{X}_{1}^{(1)}(r p+t)= & X_{1}^{(01)}(r p+1) e^{-\beta_{1}(t-1)}+p(t-2) \sum_{\xi=2}^{t} \\
& \cdot\left\{\frac{1}{2}\left[e^{-\beta_{1}(t-\xi)} f(\xi)+e^{-\beta_{1}(t-\xi+1)} f(\xi-1)\right]\right\},
\end{aligned}
$$

where the unit step function $p(t-2)$ in equation (15) is formulated by

$$
p(t-2)= \begin{cases}0, & t<2, \\ 1, & t \geq 2 .\end{cases}
$$

Unfortunately, due to the traditional $\operatorname{GMC}(1, n)$ model's poor performance, the Gaussian rule gains more popularity than Trapezoidal equivalent in recent studies for discretizing the convolution integral $[17,31]$. Consequently, the Gaussian rule is used in this paper as well. Then, the discrete response function can be presented below:

$$
\begin{aligned}
\widehat{X}_{1}^{(1)}(r p+t)= & X_{1}^{(01)}(r p+1) e^{-\beta_{1}(t-1)}+u(t-2) \sum_{\xi=2}^{t} \\
& \cdot\left\{e^{-\beta_{1}(t-\xi+(1 / 2))} * \frac{1}{2}[f(\xi)+f(\xi-1)]\right\} .
\end{aligned}
$$

where the unit step function $u(t-2)$ in equation (17) is formulated by

$$
u(t-2)= \begin{cases}0, & t<2, \\ 1, & t \geq 2 .\end{cases}
$$

For convenience, the $\operatorname{GMC}(1, n)$ model whose convolution integral is discretized by using the Gaussian rule is remarked as $\mathrm{GMC}_{\mathrm{G}}(1, n)$.
Step 6: generate the simulative and predictive values in the original domain. Through equation (14), we will get the fitted and predicted values of the $1-$ AGO sequences, which normally are considered as intermediate sequences. Subsequently, the recursive function in the original domain can be given by utilizing the 1 - IAGO method, which is defined as

$$
\begin{array}{r}
\widehat{X}_{1}^{(0)}(r p+t)=\widehat{X}_{1}^{(1)}(r p+t)-\widehat{X}_{1}^{(1)}(r p+t-1), \\
t=2,3, \ldots, r, r+1, \ldots,
\end{array}
$$

where $\widehat{X}_{1}^{(0)}(r p+t),(t=2,3, \ldots, r)$ are called simulative values, and $\widehat{X}_{1}^{(0)}(r p+t),(t \geq r+1)$ are named as predictive values.

As outlined previously, when the $u$ and $r p$ satisfy $u=r p=0$, the $\operatorname{GMC}(1, n)$ is reduced to the traditional GMC $(1, n)$ model. Moreover, when $n=1$ and $r p=0$, the $\operatorname{GMC}(1, n)$ model becomes the original $\operatorname{GMC}(1,1)$ model. Therefore, the $\operatorname{GMC}(1, n)$ model is extensions of the $\operatorname{GM}(1, n)$ and $\operatorname{GM}(1,1)$ models, indicating that the $\operatorname{GMC}(1, n)$ model has wider application fields.

2.2. Drawback Analysis of the GMC (1, n) Model. Although the popularity of $\operatorname{GMC}(1, n)$ has been increasing steadily, some inherent drawbacks still exist, which may generate unacceptable forecasting errors. After reviewing the procedures of the GMC $(1, n)$ model, we can find that two core components of this model are the model establishment and parameter estimation, which refers to equations (5) and (9), respectively. Especially for the parameter estimation, the accuracy of the estimated parameters is the precondition of obtaining the response function in equation (5). Specifically, equation (5) is used for predicting the system behavior sequence on the basis of the known parameters $\widehat{\beta}=\left[\widehat{\beta}_{1}, \widehat{\beta}_{2}, \ldots, \widehat{\beta}_{n}, \widehat{u}\right]$, which are estimated by equation (9). Hence, prior to obtaining projections, the optimal or near-optimal parameters in the $\operatorname{GMC}(1, n)$ and $\mathrm{GMC}_{\mathrm{G}}(1, n)$ models are essential for better forecasting precision, which are significantly influenced by the background value.

To expose the inherent weakness of the $\operatorname{GMC}(1, n)$ model, the actual meaning of background value needs to be further analyzed by comparing the grey differential equation in equation (9) and whitening equation in equation (5). To this end, definite integrals of equation (5) will be given on the interval $[t-1, t]$, which is displayed as

$$
\begin{gathered}
\int_{t-1}^{t} \frac{\mathrm{d} X_{1}^{(1)}(r p+\tau)}{\mathrm{d} t} \mathrm{~d} \tau+\beta_{1} \int_{t-1}^{t} X_{1}^{(1)}(r p+\tau) \mathrm{d} \tau \\
\quad=\sum_{i=2}^{n} \beta_{i} \int_{t-1}^{t} X_{i}^{(1)}(\tau) \mathrm{d} \tau+\int_{t-1}^{t} u \mathrm{~d} \tau,
\end{gathered}
$$

where 


$$
\begin{aligned}
\int_{t-1}^{t} \frac{\mathrm{d} X_{1}^{(1)}(r p+\tau)}{\mathrm{d} t} \mathrm{~d} \tau & =X_{1}^{(1)}(r p+t)-X_{1}^{(1)}(r p+t-1) \\
& =X_{1}^{(0)}(r p+t),
\end{aligned}
$$

$$
\int_{t-1}^{t} u \mathrm{~d} \tau=u
$$

Substituting equations (21) and (22) into equation (20), the discrete form of $\operatorname{GMC}(1, n)$ can be obtained as follows:

$$
\begin{gathered}
X_{1}^{(0)}(r p+t)+\beta_{1} \int_{t-1}^{t} X_{1}^{(1)}(r p+\tau) \mathrm{d} \tau \\
=\sum_{i=2}^{n} \beta_{i} \int_{t-1}^{t} X_{i}^{(1)}(\tau) \mathrm{d} \tau+u .
\end{gathered}
$$

In equation (23), the real background values in both sides of the equation are noted as
$Z_{(1)}(r p+t)=\int_{t-1}^{t} X_{1}^{(1)}(r p+\tau) \mathrm{d} \tau$ and $Z_{(i)}(t)=\int_{t-1}^{t} X_{i}^{(1)}$ $Z_{(1)}(r p+t)=\int_{t-1}^{t} X_{1}^{(1)}(r p+\tau) \mathrm{d} \tau$ and $Z_{(i)}(t)=\int_{t-1} X_{i}^{(1)}$ $(\tau) \mathrm{d} \tau$. Then, comparing equation (23) with equation (9), we find that the actual background values of $\int_{t-1}^{t} X_{1}^{(1)}(r p+t)$ and $\int_{t-1}^{t} X_{i}^{(1)}(t)$ are approximately represented by the average values of adjacent neighbor sequences in equations (7) and (8) for the $\operatorname{GMC}(1, n)$ model. However, they are not always mathematically equivalent. Accordingly, the gap between the actual background values and the approximate ones produces the inaccurate parameter estimations, which exert a negative influence on the predictive performance of the whitening equation in equation (5).

To address the weakness, Wang and Hao [23] put forward a new formula to calculate the background values based on the Mean Value Theorem of Integrals, which can be expressed as

$$
\begin{aligned}
Z_{1}^{(1)}(r p+t)= & \int_{t-1}^{t} X_{1}^{(1)}(r p+\tau) \mathrm{d} \tau=\lambda_{1} X_{1}^{(1)}(r p+t) \\
& +\left(1-\lambda_{1}\right) X_{1}^{(1)}(r p+t-1)
\end{aligned}
$$

$$
Z_{i}^{(1)}(t)=\int_{t-1}^{t} X_{i}^{(1)}(\tau) \mathrm{d} \tau \lambda_{i}=\lambda_{i} X_{i}^{(1)}(t)+\left(1-\lambda_{i}\right) X_{i}^{(1)}(t-1),
$$

where $\lambda_{i}(i=1,2, \ldots, n)$ represents the dynamic interpolation coefficients. Obviously, equations (24) and (25) are equivalent to equations (7) and (8) when $\lambda_{1}=\lambda_{2}=\cdots \lambda_{n}=0.5$, which infers that the optimized $\operatorname{GMC}(1, n)$ model having dynamic background values in [23] has better adaptability than the original one having fixed interpolation coefficients. To determine the dynamic interpolation coefficients, metaheuristic algorithms that have complex structures and require well-versed computer skills, such as Particle Swarm Algorithm and Genetic Algorithm, are necessary for combining with the $\operatorname{GMC}(1, n)$ model.

Although the optimized $\operatorname{GMC}(1, n)$ model in [23] provides an effective solution to avoid the deviations between the actual and estimated background values, potential difficulties in using this new model may increasingly arise, especially for beginners, due to the introduction of the complex optimization algorithms. Furthermore, these metaheuristic algorithms may not generate the satisfactory parameter estimations because they can fall into the local optima stagnation, consequently failing to find the actual global optimum.

Therefore, to effectively and accurately estimate the parameters in the $\operatorname{GMC}(1, n)$ model, an alternative method based on Simpson's rule is proposed for accurately measuring the background values in this paper. This algorithm, elaborated on in Section 2.3, has merits in simple structure and is easy to understand for beginners. Furthermore, it can also significantly improve the accuracy of the $\operatorname{GMC}(1, n)$ and $\mathrm{GMC}_{\mathrm{G}}(1, n)$ models, which will be demonstrated by several experiments in Section 3.

2.3. A New Optimized GMC $(1, n)$ Model Based on Simpson's Rule. In order to overcome the shortcomings of the above approaches to estimating background values, mentioned in Section 2.2, a new method based on Simpson's rule is employed to calculate the background value in the $\operatorname{GMC}(1, n)$ model. The flowchart of the new optimized $\operatorname{GMC}(1, n)$ model is displayed in Figure 1. Furthermore, the background values in $[14,23]$ are also presented in Figure 1 in comparison with the new model.

Simpson's rule, named after the English mathematician, Thomas Simpson (1710-1761), is a numerical approach to approximate the integration of a function. This method is particularly useful when integration is difficult or even impossible to do by utilizing standard techniques. The most commonly used function of Simpson's rule is provided as

$$
\int_{a}^{b} f(\tau) \mathrm{d} \tau=\frac{b-a}{6}\left[f(a)+4 f\left(\frac{a+b}{2}\right)+f(b)\right] .
$$

As introduced in Section 2.2, the actual background value can be estimated by computing the integration $\int_{t-1}^{t} X_{1}^{(1)}(r p+t)$ and $\int_{t-1}^{t} X_{i}^{(1)}(t)$, instead of the average values of adjacent neighbor sequences in equations (7) and (8). In order to be consistent with the forms of Simpson's rule in equation (26), the grey differential equation that is used for parameter estimation can be obtained by considering the definite integrals of equation (5) on the interval $[t-1, t+1]$, which is presented as

$$
\begin{aligned}
& \int_{t-1}^{t+1} \frac{\mathrm{d} X_{1}^{(1)}(r p+\tau)}{\mathrm{d} t} \mathrm{~d} \tau+\beta_{1} \int_{t-1}^{t+1} X_{1}^{(1)}(r p+\tau) \mathrm{d} \tau \\
& =\sum_{i=2}^{n} \beta_{i} \int_{t-1}^{t+1} X_{i}^{(1)}(\tau) \mathrm{d} \tau+\int_{t-1}^{t+1} u \mathrm{~d} \tau,
\end{aligned}
$$

where

$$
\int_{t-1}^{t+1} \frac{\mathrm{d} X_{1}^{(1)}(r p+\tau)}{\mathrm{d} t} \mathrm{~d} \tau=X_{1}^{(1)}(r p+t+1)-X_{1}^{(1)}(r p+t-1)
$$

$$
\int_{t-1}^{t+1} u \mathrm{~d} \tau=2 u
$$



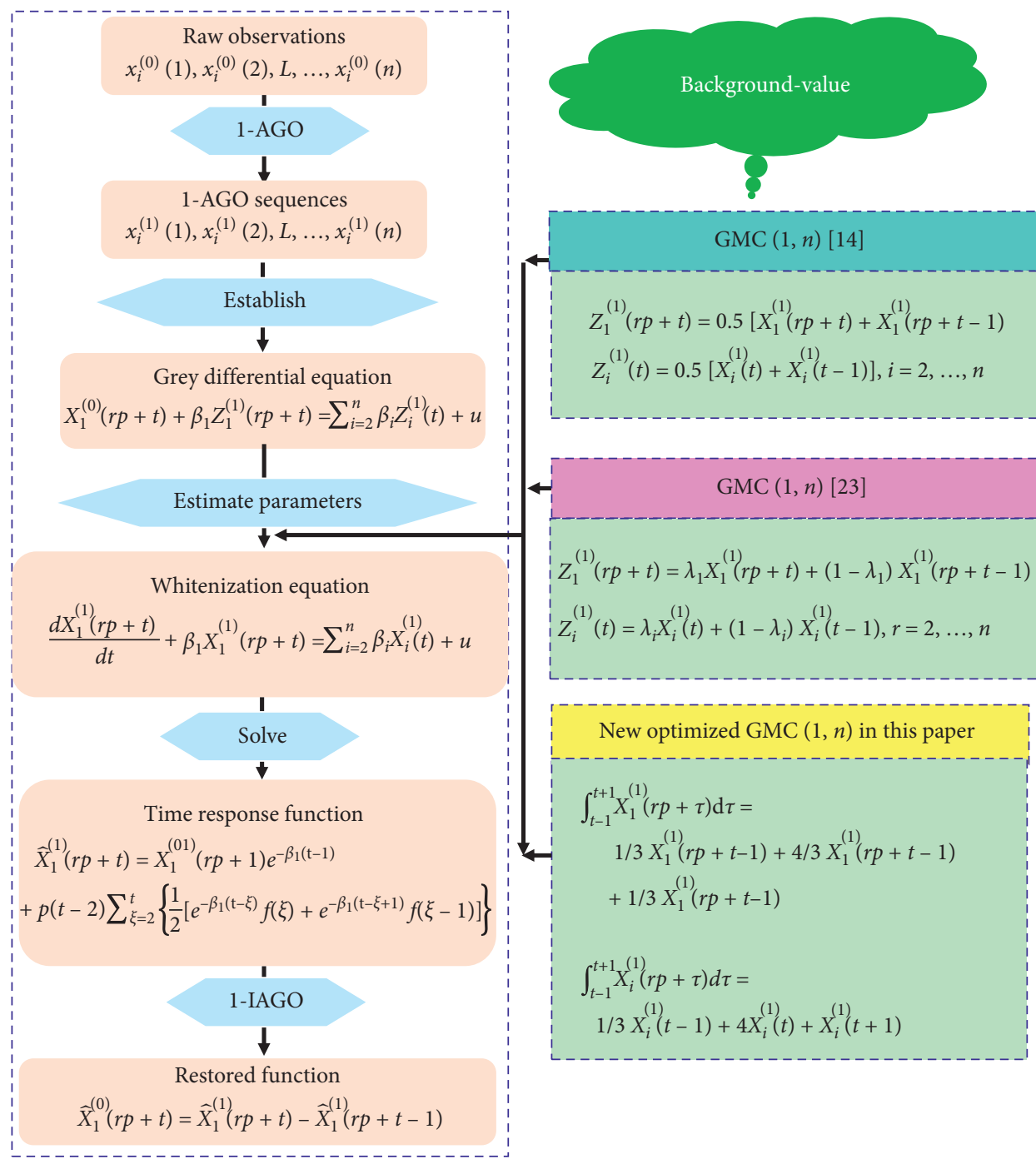

FIGURE 1: Flowchart of the GMC $(1, n)$ model with different approaches to estimating background values.

Hence, substituting equations (28) and (29) into equation (27), the grey differential equation is mathematically equivalent to

$$
\begin{aligned}
& X_{1}^{(1)}(r p+t+1)-X_{1}^{(1)}(r p+t-1)+\beta_{1} \int_{t-1}^{t+1} X_{1}^{(1)}(r p+\tau) \mathrm{d} \tau \\
& \quad=\sum_{i=2}^{n} \beta_{i} \int_{t-1}^{t+1} X_{i}^{(1)}(\tau) \mathrm{d} \tau+2 u .
\end{aligned}
$$

According to Simpson's rule in equation (26), we can get

$$
\begin{aligned}
\int_{t-1}^{t+1} X_{1}^{(1)}(r p+\tau) \mathrm{d} \tau= & \frac{1}{3}\left[X_{1}^{(1)}(r p+t-1)+4 X_{1}^{(1)}\right. \\
& \left.\cdot(r p+t-1)+X_{1}^{(1)}(r p+t+1)\right],
\end{aligned}
$$

$\int_{t-1}^{t+1} X_{i}^{(1)}(r p+\tau) \mathrm{d} \tau=\frac{1}{3}\left[X_{i}^{(1)}(t-1)+4 X_{i}^{(1)}(t)+X_{i}^{(1)}(t+1)\right]$.
Substituting and simplifying, equation (30) mathematically equals to

$$
\begin{aligned}
X_{1}^{(1)} & (r p+t+1)-X_{1}^{(1)}(r p+t-1)+\frac{\beta_{1}}{3}\left[X_{1}^{(1)}(r p+t-1)\right. \\
& \left.+4 X_{1}^{(1)}(r p+t-1)+X_{1}^{(1)}(r p+t+1)\right] \\
= & \sum_{i=2}^{n} \frac{\beta_{i}}{3}\left[X_{i}^{(1)}(t-1)+4 X_{i}^{(1)}(t)+X_{i}^{(1)}(t+1)\right]+2 u .
\end{aligned}
$$

Simplifying, we can get this:

$$
\begin{gathered}
-\frac{\beta_{1}}{3}\left[X_{1}^{(1)}(r p+t-1)+4 X_{1}^{(1)}(r p+t-1)+X_{1}^{(1)}(r p+t+1)\right] \\
+\sum_{i=2}^{n} \frac{\beta_{i}}{3}\left[X_{i}^{(1)}(t-1)+4 X_{i}^{(1)}(t)+X_{i}^{(1)}(t+1)\right]+2 u \\
=X_{1}^{(0)}(r p+t+1)+X_{1}^{(0)}(r p+t), t=2,3, \ldots, r-1 .
\end{gathered}
$$


Assume that

$$
\left\{\begin{array}{l}
m_{t, 1}=-\frac{1}{3}\left[X_{1}^{(1)}(r p+t-1)+4 X_{1}^{(1)}(r p+t-1)+X_{1}^{(1)}(r p+t+1)\right] \\
m_{t, i}=\frac{1}{3}\left[X_{i}^{(1)}(t-1)+4 X_{i}^{(1)}(t)+X_{i}^{(1)}(t+1)\right], \quad i=2,3, \ldots, n, \\
d_{t}=X_{1}^{(0)}(r p+t+1)+X_{1}^{(0)}(r p+t) .
\end{array}\right.
$$

Subsequently, substituting equation (35) and the values of $t$ into equation (34), we can obtain

$$
\left\{\begin{array}{l}
\beta_{1} m_{2,1}+\beta_{2} m_{2,2}+\beta_{3} m_{2,3}+\cdots+\beta_{n} m_{2, n}+2 u=d_{2} \\
\beta_{1} m_{3,1}+\beta_{2} m_{3,2}+\beta_{3} m_{3,3}+\cdots+\beta_{n} m_{3, n}+2 u=d_{3} \\
\cdots \quad \cdots \quad \cdots \quad \cdots \\
\beta_{1} m_{r-1,1}+\beta_{2} m_{r-1,2}+\beta_{3} m_{r-1,3}+\cdots+\beta_{n} m_{r-1, n}+2 u=d_{r-1} .
\end{array}\right.
$$

Equation (36) can be written into the matrix form $M=D \widehat{\beta}$, where

$$
\begin{aligned}
M & =\left[\begin{array}{c}
d_{2} \\
d_{3} \\
\vdots \\
d_{r-1}
\end{array}\right], \\
D & =\left[\begin{array}{ccccc}
m_{2,1} & m_{2,2} & \cdots & m_{2, n} & 2 \\
m_{3,1} & m_{3,2} & \cdots & m_{3,3} & 2 \\
\vdots & \vdots & \ddots & \vdots & \vdots \\
m_{r-1,1} & m_{r-1,2} & \cdots & m_{r-1, n} & 2
\end{array}\right] .
\end{aligned}
$$

Solving this above matrix, the estimated values of parameters will be formulated:

$$
\widehat{\beta}=\left[\widehat{\beta}_{1}, \widehat{\beta}_{2}, \ldots, \widehat{\beta}_{n}, \widehat{u}\right]=\left(D^{T} D\right)^{-1} D^{T} M .
$$

Similar to the $\operatorname{GMC}(1, n)$ and $\operatorname{GMC}_{\mathrm{G}}(1, n)$ models, we can also achieve two optimized models by using the Trapezoidal and Gaussian rule to discretize the convolution integral in equation (13). Specifically, substituting the parameter values $\widehat{\beta}=\left[\widehat{\beta}_{1}, \widehat{\beta}_{2}, \ldots, \widehat{\beta}_{n}, \widehat{u}\right]$ into equations (14) and (17), the discrete response function can be obtained:

$$
\begin{aligned}
& \widehat{X}_{1}^{(1)}(r p+t)= X_{1}^{(0)}(r p+1) e^{-\beta_{1}(t-1)}+u(t-2) \sum_{\xi=2}^{t} \\
& \cdot\left\{e^{-\beta_{1}(t-\xi+(1 / 2))} * \frac{1}{2}[f(\xi)+f(\xi-1)]\right\}, \\
& \widehat{X}_{1}^{(1)}(r p+t)=X_{1}^{(0)}(r p+1) e^{-\beta_{1}(t-1)}+u(t-2) \sum_{\xi=2}^{t} \\
& \cdot\left\{\frac{1}{2}\left[e^{-\beta_{1}(t-\xi)} f(\xi)+e^{-\beta_{1}(t-\xi+1)} f(\xi-1)\right]\right\},
\end{aligned}
$$

Where the unit step function $u(t-2)$ and function $f(t)$ in equations (39) and (40) are formulated by

$$
\begin{aligned}
u(t-2) & = \begin{cases}0, & t<2, \\
1, & t \geq 2,\end{cases} \\
f(t) & =\sum_{i=2}^{n} \beta_{i} X_{i}^{(1)}(t)+u .
\end{aligned}
$$

For convenience, these two models in (39) and (40) are remarked as $\operatorname{OGMC}(1, n)$ and $\operatorname{OGMC}_{\mathrm{G}}(1, n)$ models, respectively. Subsequently, by utilizing the 1 - IAGO method, the recursive function in the original domain is defined as

$$
\begin{array}{r}
\widehat{X}_{1}^{(0)}(r p+t)=\widehat{X}_{1}^{(1)}(r p+t)-\widehat{X}_{1}^{(1)}(r p+t-1), \\
t=2,3, \ldots, r, r+1, \ldots,
\end{array}
$$

Where $\widehat{X}_{1}^{(0)}(r p+t),(t=2,3, \ldots, r)$ are called simulative values, and $\widehat{X}_{1}^{(0)}(r p+t),(t \geq r+1)$ are named as predictive values.

Based on Simpson's rule, the background values of the GMC $(1, n)$ model can be accurately calculated, which is quite different from those in $[14,23]$. Moreover, Figure 1 provides the detailed procedures of the optimized model, which is helpful for beginners to use this model.

2.4. Performance Evaluation. In addition to the modeling procedures of the $\operatorname{GMC}(1, n)$ model, the measuring indicators are also essential for evaluating the model's performance. In this study, three different indices will be adopted to examine the performance of competing models, including APE (Absolute Percentage Error), MAPE (Mean Absolute Percentage Error), and RMSE (Root Mean Squared Error).

The algorithms of the three indices are calculated using the following equations:

$$
\begin{aligned}
\mathrm{APE} & =\frac{\left|\hat{x}^{(0)}(t)-x^{(0)}(t)\right|}{x^{(0)}(t)} \times 100 \%, \quad t=2,3, \ldots, n, n+1, \ldots, \\
\mathrm{MAPE} & =\frac{1}{n-1} \sum_{t=2}^{n} \operatorname{APE}(t), \\
\mathrm{RMSE} & =\sqrt{\frac{1}{n-1} \sum_{t=2}^{n}\left(\hat{x}^{(0)}(t)-x^{(0)}(t)\right)^{2}},
\end{aligned}
$$

where $x^{(0)}(t)$ is the actual value at time $t$, and the $\widehat{x}^{(0)}(t)$ is the corresponding simulative or predictive value at time $t$. 


\section{Experiments and Analysis}

In this section, for the sake of demonstrating the practicability and efficacy of the new optimized model, three empirical studies are carried out in comparison with traditional multivariable grey prediction models.

3.1. Case One: Predicting the Tensile Strength of a Material. The multivariable grey prediction models, such as $\operatorname{GM}(1,2)$ and $\operatorname{GMC}(1,2)$, can be used for the indirect measurement of the tensile strength of a material [14], the efficacy of the $\operatorname{OGMC}(1,2)$ and $\operatorname{OGMC}_{\mathrm{G}}(1,2)$ models can be revealed by comparing the experimental results of predicting tensile strength of a material in comparison with the $\operatorname{GMC}(1,2)$, $\mathrm{GMC}_{\mathrm{G}}(1,2), \operatorname{GM}(1,2)$, and $\operatorname{DGM}(1,2)$ models. The original data are presented in Table 1, which were collected from the experimental observations in Table 117 of Samuel [33]. In addition, the tensile strength represents the system behavior sequence $\left(X_{1}^{(0)}\right)$, and the Brinell hardness works as the relevant series $\left(X_{2}^{(0)}\right)$. Moreover, six data points of $X_{1}^{(0)}$ and $X_{2}^{(0)}$ are utilized for model calibration, and the remaining four observations of $X_{1}^{(0)}$ are used for checking the predictive performance, namely, setting parameters $n=2, r=6, r p=0$, and $r f=4$ [33].

As introduced in Sections 2.1 and 2.3, the procedures and flowchart of $\operatorname{GMC}(1,2)$ and its extensions are presented, and the procedures of $\operatorname{GM}(1,2)$ and $\operatorname{DGM}(1,2)$ are similar to those of $\operatorname{GMC}(1,2)$. Thus, according to these procedures, the estimated parameter-values of six competing models are given in Table 2.

Taking $\operatorname{OGMC}(1,2)$ as an example for explanations, we can obtain the parameter values $\widehat{\beta}=\left[\widehat{\beta}_{1}, \widehat{\beta}_{2}, \widehat{u}\right]=$ $[0.15387,0.28354,885.67276]$ after substituting the original data into equations (35) and (36). Subsequently, substituting the parameter values into equation (39), we will have the discrete response function:

$$
\begin{aligned}
\widehat{X}_{1}^{(1)}(t)= & X_{1}^{(0)}(1) e^{-0.15387(t-1)}+u(t-2) \sum_{\xi=2}^{t} \\
& \cdot\left\{e^{-0.15387(t-\xi+(1 / 2))} * \frac{1}{2}[f(\xi)+f(\xi-1)]\right\},
\end{aligned}
$$

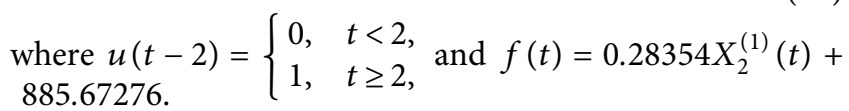

By using the time response function in equation (44), the forecasted results and the values of three indices measuring accuracy will be obtained, which are shown in Table 3 . It is obvious that four $\operatorname{GMC}(1,2)$ and its extensions are superior to the traditional $\operatorname{GM}(1,2)$ and $\operatorname{DGM}(1,2)$ whose MAPE values reach $24.94 \%$ and $55.91 \%$, respectively. The reason for this phenomenon is that $\operatorname{GM}(1,2)$ has an incorrect time response function and $\operatorname{DGM}(1,2)$ has narrow application fields. In contrast, the $\operatorname{GMC}(1,2)$ and $\operatorname{GMC}_{\mathrm{G}}(1,2)$ models are able to provide accurate forecasts because their modeling values with convolution integral are exact theoretically [14]. However, the $\operatorname{OGMC}(1,2)$ and $\operatorname{OGMC}_{\mathrm{G}}(1,2)$ models seem to obtain a litter better forecasting performance owing to smaller MAPE values, 2.48 and 2.52, respectively. Furthermore, the RMSE values of these two models are much smaller than the other four competitors, inferring good stability and reliability of the optimized background values based on Simpson's rule.

In general, the $\operatorname{GMC}(1,2), \operatorname{GMC}_{\mathrm{G}}(1,2), \operatorname{OGMC}(1,2)$, and $\mathrm{OGMC}_{\mathrm{G}}(1,2)$ models share almost the similar precision in predicting the tensile strength, and they are equally applicable for further estimations. Additionally, the optimized background values based on Simpson's rule can effectively contribute to the improvements of forecasting precision.

3.2. Case Two: Estimating the Gross Industrial Output Value. In addition to the numerical example from Tien's study [14], another actual case concerning forecasting China's industrial output value, collected from Ma and Liu [31], is selected to test the effectiveness and practicability of the proposed models. The raw observations, listed in Table 4, are collected from the National Bureau of Statistics of China, who published correct, official, and complete data. Apparently, the industrial output value is the system behavior sequence, marked as $X_{1}^{(0)}$. Then, the total current assets and fix assets represent the relevant series, noted as $X_{2}^{(0)}$ and $X_{3}^{(0)}$. The $\operatorname{GMC}(1,3), \mathrm{GMC}_{\mathrm{G}}(1,3), \quad \operatorname{OGMC}(1,3), \quad \operatorname{OGMC}_{\mathrm{G}}(1,3)$, $\operatorname{GM}(1,3)$, and $\operatorname{DGM}(1,3)$ are employed for forecasting China's industrial output value with parameter setting $m=3, r=10, r p=0$, and $r f=3$. In other words, ten data points of the three variables are used for estimating parameters, and the last three observations of the system behavior series are selected for testing the predictive performance.

Taking the OGMC $(1,3)$ model as an example for explanations, we can obtain the parameter values $\widehat{\beta}=\left[\widehat{\beta}_{1}, \widehat{\beta}_{2}, \widehat{\beta}_{3}, \widehat{u}\right]=[0.53849,5.36851,-2.79249,36418.02856]$ by utilizing equations (27)-(36). Subsequently, substituting the parameter values into equation (39), the discrete response function will be given:

$$
\begin{aligned}
\widehat{X}_{1}^{(1)}(t)= & X_{1}^{(0)}(1) e^{-0.53849(t-1)}+u(t-2) \sum_{\xi=2}^{t} \\
& \cdot\left\{e^{-0.53849(t-\xi+(1 / 2))} * \frac{1}{2}[f(\xi)+f(\xi-1)]\right\},
\end{aligned}
$$

where $u(t-2)=\left\{\begin{array}{ll}0, & t<2, \\ 1, & t \geq 2,\end{array}\right.$ and $f(t)=0.5 .36851 X_{2}^{(1)}(t)-$ $2.79249 X_{2}^{(1)}(t)+36418.02856$.

Additionally, the parameter values of the other five competing models are also can be estimated, which is listed in Table 5.

Subsequently, by using the discrete response function, the forecasted results and values of the evaluation indicators will be obtained, which are displayed in Table 6. As this table illustrates, similar conclusions can be achieved: the $\operatorname{GMC}(1,3), \mathrm{GMC}_{\mathrm{G}}(1,3), \operatorname{OGMC}(1,3)$, and $\operatorname{OGMC}_{\mathrm{G}}(1,3)$ models significantly outperform the $\operatorname{GM}(1,3)$ and 
TABLE 1: Original data in case one.

\begin{tabular}{lcc}
\hline$i$ (temperature) & Tensile strength $X_{1}^{(0)}(\mathrm{MPa})$ & Brinell hardness $X_{2}^{(0)}(\mathrm{HBW})$ \\
\hline $1\left(400^{\circ} \mathrm{F}\right)$ & 897 & 514 \\
$2\left(500^{\circ} \mathrm{F}\right)$ & 897 & 495 \\
$3\left(600^{\circ} \mathrm{F}\right)$ & 890 & 444 \\
$4\left(700^{\circ} \mathrm{F}\right)$ & 876 & 401 \\
$5\left(800^{\circ} \mathrm{F}\right)$ & 848 & 352 \\
$6\left(900^{\circ} \mathrm{F}\right)$ & 814 & 293 \\
$7\left(1000^{\circ} \mathrm{F}\right)$ & 779 & 269 \\
$8\left(1100^{\circ} \mathrm{F}\right)$ & 738 & 235 \\
$9\left(1200^{\circ} \mathrm{F}\right)$ & 669 & 201 \\
$10\left(1300^{\circ} \mathrm{F}\right)$ & 600 & 187 \\
\hline
\end{tabular}

TABLE 2: Estimated parameter values of six competing models in case one.

\begin{tabular}{lcccccc}
\hline Parameters & GMC $(1,2)$ & OGMC $(1,2)$ & GMC $_{\mathrm{G}}(1,2)$ & OGMC $_{\mathrm{G}}(1,2)$ & $\mathrm{GM}(1,2)$ & $\mathrm{DGM}(1,2)$ \\
\hline$\widehat{\beta}_{1}$ & 0.14378 & 0.15387 & 0.14378 & 0.15387 & 0.84617 & -0.86519 \\
$\widehat{\beta}_{2}$ & 0.26139 & 0.28354 & 0.26139 & 0.28354 & 1.93150 & 0.25951 \\
$\widehat{u}$ & 891.16435 & 885.67276 & 891.16435 & 885.67276 & - & 755.74471 \\
\hline
\end{tabular}

TABLE 3: The forecasted results and the values of three indices measuring accuracy by six competing models in case one.

\begin{tabular}{|c|c|c|c|c|c|c|c|}
\hline \multicolumn{2}{|c|}{ Original values } & 779 & 738 & 669 & 600 & MAPE & RMSE \\
\hline \multirow{2}{*}{$\operatorname{GMC}(1,2)$} & Forecasted values & 774.67 & 732.08 & 686.91 & 642.11 & \multirow{2}{*}{2.76} & \multirow{2}{*}{23.17} \\
\hline & Error (\%) & 0.56 & 0.80 & 2.68 & 7.02 & & \\
\hline \multirow{2}{*}{$\operatorname{OGMC}(1,2)$} & Forecasted values & 771.98 & 727.90 & 681.15 & 634.95 & \multirow{2}{*}{2.48} & \multirow{2}{*}{19.50} \\
\hline & Error (\%) & 0.90 & 1.37 & 1.82 & 5.83 & & \\
\hline \multirow{2}{*}{$\mathrm{GMC}_{\mathrm{G}}(1,2)$} & Forecasted values & 771.88 & 729.81 & 685.10 & 640.54 & \multirow{2}{*}{2.80} & \multirow{2}{*}{22.48} \\
\hline & Error (\%) & 0.91 & 1.11 & 2.41 & 6.76 & & \\
\hline \multirow{2}{*}{$\mathrm{OGMC}_{\mathrm{G}}(1,2)$} & Forecasted values & 768.88 & 725.39 & 679.18 & 633.25 & \multirow{2}{*}{2.52} & \multirow{2}{*}{19.17} \\
\hline & Error (\%) & 1.30 & 1.71 & 1.52 & 5.54 & & \\
\hline \multirow{2}{*}{$\mathrm{GM}(1,2)$} & Forecasted values & 650.10 & 554.29 & 467.39 & 430.85 & \multirow{2}{*}{24.94} & \multirow{2}{*}{172.93} \\
\hline & Error (\%) & 16.55 & 24.89 & 30.14 & 28.19 & & \\
\hline \multirow{2}{*}{$\operatorname{DGM}(1,2)$} & Forecasted values & 377.57 & 327.26 & 282.54 & 247.85 & \multirow{2}{*}{55.91} & \multirow{2}{*}{388.34} \\
\hline & Error (\%) & 51.53 & 55.66 & 57.77 & 58.69 & & \\
\hline
\end{tabular}

TABLE 4: Original data in case two (unit: $10^{4}$ yuan) [31].

\begin{tabular}{lccc}
\hline$i$ (year) & $\begin{array}{c}\text { Industrial output values } \\
X_{1}^{(0)}\end{array}$ & $\begin{array}{c}\text { Total current assets } \\
X_{2}^{(0)}\end{array}$ & $\begin{array}{c}\text { Fix assets } \\
X_{3}^{(0)}\end{array}$ \\
\hline 1999 & 72707.04 & 49630.23 & 71847.09 \\
2000 & 85673.66 & 54338.15 & 78646.30 \\
2001 & 95448.98 & 57804.97 & 86293.10 \\
2002 & 110776.48 & 63468.46 & 93887.95 \\
2003 & 142271.22 & 76163.74 & 105557.09 \\
2004 & 201722.19 & 97183.74 & 125761.85 \\
2005 & 251619.50 & 111031.41 & 143143.63 \\
2006 & 316588.96 & 132310.12 & 168850.20 \\
2007 & 405177.13 & 163259.62 & 198739.27 \\
2008 & 507284.89 & 195681.75 & 245352.80 \\
2009 & 548311.42 & 223038.68 & 278541.09 \\
2010 & 698590.42 & 279227.32 & 334839.41 \\
2011 & 844268.79 & 327778.65 & 386086.72 \\
\hline
\end{tabular}

$\operatorname{DGM}(1,3)$ models. Moreover, the MAPE values of the $\operatorname{GM}(1,3)$ and $\operatorname{DGM}(1,3)$ models are $26.52 \%$ and $46.22 \%$, which means these two models are unable to forecast future values of China's industrial output values due to their bad performances. On the other hand, the grey prediction models with convolution integral are able to provide very competitive results in terms of improved accuracy.

As anticipated, with its 5.4\%MAPE values and 39155.02RMSE values, the $\mathrm{OGMC}_{\mathrm{G}}(1,3)$ model provides the highest prediction accuracy followed by $\operatorname{GMC}_{\mathrm{G}}(1,3)$, $\mathrm{OGMC}_{\mathrm{G}}(1,3)$, and $\operatorname{GMC}(1,3)$, respectively. The reasons for such an excellent performance of this optimized model are mainly provided from two perspectives. On one hand, the Gaussian rule that discretizing the convolution interval is generally more accurate than the Trapezoid rule, which can also be verified by other studies, such as Ma and Liu [31], Tien [15], and Wang [18]. On the other hand, the improvements of the background values based on Simpson's rule are equally important to enhancing its forecasting capability, because the optimized background values are crucial requirement to ensure the reliable and accurate parameter estimations, explained in Section 2.2. Similar findings can also be obtained from the comparison of the $\operatorname{GMC}(1,3)$ and $\operatorname{OGMC}(1,3)$ models, of which the accuracy rate is heavily dependent on the optimization of background values. 
TABLE 5: Estimated parameter values of six competing models in case two.

\begin{tabular}{lcccccc}
\hline Parameters & $\mathrm{GMC}(1,3)$ & $\mathrm{OGMC}(1,3)$ & $\mathrm{GMC}_{\mathrm{G}}(1,3)$ & $\mathrm{OGMC}_{\mathrm{G}}(1,3)$ & $\mathrm{GM}(1,3)$ & $\mathrm{DGM}(1,3)$ \\
\hline$\widehat{\beta}_{1}$ & 0.56111 & 0.53849 & 0.56111 & 0.53849 & 0.62073 & -0.60170 \\
$\widehat{\beta}_{2}$ & 5.79185 & 5.36851 & 5.79185 & 5.36851 & 5.01229 & 3.72981 \\
$\widehat{\beta}_{3}$ & -3.07901 & -2.79249 & -3.07901 & -2.79249 & -2.51896 & -1.96757 \\
$\widehat{u}$ & 68074.74553 & 36418.02856 & 68074.74553 & 36418.02856 & - & 22097.40372 \\
\hline
\end{tabular}

TABLE 6: The forecasted results and the values of three indices measuring accuracy by six competing models in case two.

\begin{tabular}{|c|c|c|c|c|c|c|}
\hline \multicolumn{2}{|c|}{ Original values } & 548311.42 & 698590.42 & 844268.79 & MAPE & RMSE \\
\hline $\operatorname{GMC}(1,3)$ & $\begin{array}{l}\text { Forecasted values } \\
\text { Error (\%) }\end{array}$ & $\begin{array}{c}626326.04 \\
14.23\end{array}$ & $\begin{array}{c}774366.43 \\
10.85\end{array}$ & $\begin{array}{c}963930.75 \\
14.17\end{array}$ & 13.08 & 93358.22 \\
\hline $\operatorname{OGMC}(1,3)$ & $\begin{array}{l}\text { Forecasted values } \\
\text { Error (\%) }\end{array}$ & $\begin{array}{c}622548.58 \\
13.54\end{array}$ & $\begin{array}{c}767770.55 \\
9.90\end{array}$ & $\begin{array}{c}953445.29 \\
12.93\end{array}$ & 12.12 & 86055.32 \\
\hline $\operatorname{GMC}_{\mathrm{G}}(1,3)$ & $\begin{array}{l}\text { Forecasted values } \\
\text { Error (\%) }\end{array}$ & $\begin{array}{l}589395.96 \\
7.49\end{array}$ & $\begin{array}{c}721699.65 \\
3.31\end{array}$ & $\begin{array}{c}901242.19 \\
6.75\end{array}$ & 5.85 & 42692.48 \\
\hline $\mathrm{OGMC}_{\mathrm{G}}(1,3)$ & $\begin{array}{l}\text { Forecasted values } \\
\text { Error (\%) }\end{array}$ & $\begin{array}{l}588046.17 \\
7.25\end{array}$ & $\begin{array}{c}718898.95 \\
2.91\end{array}$ & $\begin{array}{c}895337.97 \\
6.05\end{array}$ & 5.40 & 39155.02 \\
\hline $\operatorname{GM}(1,3)$ & $\begin{array}{l}\text { Forecasted values } \\
\text { Error (\%) }\end{array}$ & $\begin{array}{c}673916.59 \\
22.91\end{array}$ & $\begin{array}{c}898040.07 \\
28.55\end{array}$ & $\begin{array}{c}1081472.75 \\
28.10\end{array}$ & 26.52 & 193065.22 \\
\hline $\operatorname{DGM}(1,3)$ & $\begin{array}{l}\text { Forecasted values } \\
\text { Error (\%) }\end{array}$ & $\begin{array}{c}283772.70 \\
48.25\end{array}$ & $\begin{array}{c}382603.22 \\
45.23\end{array}$ & $\begin{array}{c}462874.97 \\
45.17\end{array}$ & 46.22 & 324186.11 \\
\hline
\end{tabular}

Generally, the $\mathrm{OGMC}_{\mathrm{G}}(1,3)$ model obtains the highest forecasting ability leading itself as the optimal technique for the projections of China's industrial output value. Moreover, Simpson's rule can be employed for calculating the background values with high precision, which is essential for parameter estimations.

3.3. Case Three: Forecasting Electronic Waste in Washington State. The empirical results of cases one and two demonstrate the superior forecasting performance of the $\operatorname{OGMC}(1, n)$ and $\mathrm{OGMC}_{\mathrm{G}}(1, n)$ models having optimized background values based on Simpson's rule, in comparison with other competitors. Furthermore, the $\operatorname{OGMC}_{\mathrm{G}}(1, n)$ model achieves a litter higher accuracy than the $\operatorname{OGMC}(1, n)$ model. Therefore, in addition to the above two examples coming from the published papers, another new case concerning electronic waste is introduced to verify the proposed model's efficacy and reliability in the following subsections.

3.3.1. Data Description and Model Calibration. As introduced in earlier work $[34,35]$, e-waste has increasingly become one of the major contributors of municipal solid waste (MSW), whose commonly used influencing factors are the population density and median household income. In this paper, the original data sets of e-waste from 2003 to 2015 are available from the government website (ecology.wa.gov), whereas data since 2016 are unavailable. In addition, observations of these two factors are collected from the Washington State Office of Financial Management website $[36,37]$. All the available data of these three variables are presented in Table 7. Moreover, in Table 7, data sets from 2003 to 2012 are used for establishing six models, whereas observations from 2013 to 2015 are employed for comparison. In the GMC $(1, n)$ and its extensions, the parameters are used as $m=3, r=10, r p=0$, and $r f=3$.

All computations are executed on MATLAB 2016a and the parameter estimations are given in Table 8 . Then, we take the OGMC $(1,3)$ model as an example for explaining the main procedures. By using equations (1)-(4) and (27)-(36), we can obtain the estimated parameters, namely, $\widehat{\beta}=\left[\widehat{\beta}_{1}, \widehat{\beta}_{2}\right.$, $\left.\widehat{\beta}_{3}, \widehat{u}\right]=[0.31662,-420.77848,1.19678,9020.93243]$. Furthermore, the discrete response function will be displayed below:

$$
\begin{aligned}
\widehat{X}_{1}^{(1)}(t)= & X_{1}^{(0)}(1) e^{-0.31662(t-1)}+u(t-2) \sum_{\xi=2}^{t} \\
& \cdot\left\{e^{-0.31662(t-\xi+(1 / 2))} * \frac{1}{2}[f(\xi)+f(\xi-1)]\right\},
\end{aligned}
$$

where $u(t-2)=\left\{\begin{array}{ll}0, & t<2, \\ 1, & t \geq 2,\end{array}\right.$ and $f(t)=-420.77848 X_{2}^{(1)}$ $(t) 1.19678 X_{2}^{(1)}(t)+9020.93243$. Subsequently, the visual comparison of original and forecasted $\operatorname{OGMC}(1,3)$ values is shown in Table 9.

3.3.2. Comparative Analysis of the Forecasting Performance by Six Competing Models. As seen in Table 9, notice that $\operatorname{GM}(1,3)$ and $\operatorname{GM}(1,3)$ produce inaccurate results with $42.55 \%$ and $68.51 \%$ MAPE values, respectively. Hence, these two models fail to predict the e-waste amount. In contrast, the $\operatorname{GMC}(1,3)$ and $\mathrm{GMC}_{\mathrm{G}}(1,3)$ models, as expected, provide obvious improvements in the forecasting precision. The reason for this situation is that the modeling values by using conventual integral in these two models are theoretically correct, while those in the $\operatorname{GM}(1,3)$ model are wrong [8]. In 
TABle 7: E-waste, population density, and median household income in case three.

\begin{tabular}{lccc}
\hline$i$ (year) & $\begin{array}{c}\text { E-waste } \\
X_{1}^{(0)}(\text { tons })\end{array}$ & $\begin{array}{c}\text { Population density } \\
X_{2}^{(0)}(\text { people/se mi) }\end{array}$ & $\begin{array}{c}\text { Median household } \\
\text { Income } X_{3}^{(0)}(\$)\end{array}$ \\
\hline 2003 & 18108.19 & 92.07 & 46967.25 \\
2004 & 27341.56 & 93.30 & 49585.35 \\
2005 & 35887.90 & 94.66 & 50004.20 \\
2006 & 46126.41 & 96.48 & 53522.31 \\
2007 & 53737.51 & 98.06 & 56141.32 \\
2008 & 62071.46 & 99.31 & 57857.52 \\
2009 & 69246.27 & 100.27 & 55458.05 \\
2010 & 68777.91 & 101.19 & 54888.45 \\
2011 & 69673.02 & 101.84 & 55500.26 \\
2012 & 73851.24 & 102.59 & 56443.76 \\
2013 & 65894.78 & 103.56 & 57283.94 \\
2014 & 67822.93 & 104.85 & 60153.00 \\
2015 & 72103.41 & 106.26 & 63439.20 \\
\hline
\end{tabular}

TABLE 8: Estimated parameter values of six competing models in case three.

\begin{tabular}{lcccccc}
\hline Parameters & GMC $(1,3)$ & OGMC $(1,3)$ & $\mathrm{GMC}_{\mathrm{G}}(1,3)$ & $\mathrm{OGMC}_{\mathrm{G}}(1,3)$ & $\mathrm{GM}(1,3)$ & $\mathrm{DGM}(1,3)$ \\
\hline$\widehat{\beta}_{1}$ & 0.30047 & 0.31662 & 0.30047 & 0.31662 & 0.27732 & -0.76644 \\
$\widehat{\beta}_{2}$ & -455.74524 & -420.77848 & -455.74524 & -420.77848 & -273.98465 & -405.07032 \\
$\widehat{\beta}_{3}$ & 1.24313 & 1.19678 & 1.24313 & 1.19678 & 0.88929 & 1.06417 \\
$\widehat{u}$ & 10694.07636 & 9020.93243 & 10694.07636 & 9020.93243 & - & 3963.42238 \\
\hline
\end{tabular}

TABLE 9: The forecasted results and the values of three indices measuring accuracy by six competing models in case three.

\begin{tabular}{|c|c|c|c|c|c|c|}
\hline \multicolumn{2}{|c|}{ Original values } & \multirow{3}{*}{$\begin{array}{c}65894.78 \\
74781.44 \\
13.49\end{array}$} & \multirow{3}{*}{$\begin{array}{c}67822.93 \\
77760.78 \\
14.65\end{array}$} & \multirow{3}{*}{$\begin{array}{c}72103.41 \\
82791.00 \\
14.82\end{array}$} & \multirow{3}{*}{$\begin{array}{c}\text { MAPE } \\
14.32\end{array}$} & \multirow{3}{*}{$\begin{array}{c}\text { RMSE } \\
9865.06\end{array}$} \\
\hline & Forecasted values & & & & & \\
\hline $\operatorname{GMC}(1,3)$ & Error (\%) & & & & & \\
\hline \multirow{2}{*}{$\operatorname{OGMC}(1,3)$} & Forecasted values & 74387.88 & 77235.75 & 82033.66 & \multirow{2}{*}{13.51} & \multirow{2}{*}{9297.73} \\
\hline & Error (\%) & 12.89 & 13.88 & 13.77 & & \\
\hline \multirow{2}{*}{$\mathrm{GMC}_{\mathrm{G}}(1,3)$} & Forecasted values & 73784.29 & 76581.01 & 81414.67 & \multirow{2}{*}{12.60} & \multirow{2}{*}{8672.71} \\
\hline & Error (\%) & 11.97 & 12.91 & 12.91 & & \\
\hline \multirow{2}{*}{$\operatorname{OGMC}_{\mathrm{G}}(1,3)$} & Forecasted values & 73306.88 & 75968.74 & 80567.28 & \multirow{2}{*}{11.67} & \multirow{2}{*}{8019.37} \\
\hline & Error (\%) & 12.01 & 12.01 & 11.74 & & \\
\hline \multirow{2}{*}{$\operatorname{GM}(1,3)$} & Forecasted values & 90944.46 & 97409.07 & 105286.45 & \multirow{2}{*}{42.55} & \multirow{2}{*}{29461.50} \\
\hline & Error (\%) & 38.01 & 43.62 & 46.02 & & \\
\hline \multirow{2}{*}{$\operatorname{DGM}(1,3)$} & Forecasted values & 18986.34 & 21522.63 & 24452.89 & \multirow{2}{*}{68.51} & \multirow{2}{*}{46956.3} \\
\hline & Error (\%) & 71.19 & 68.27 & 66.09 & & \\
\hline
\end{tabular}

addition, the Gaussian rule performs a little better than the Trapezoid rule because the $\mathrm{GMC}_{\mathrm{G}}(1,3)$ model achieves higher predicting accuracy.

Compared with the previous $\operatorname{GMC}(1,3)$ and $\operatorname{GMC}_{\mathrm{G}}(1,3)$ models, the $\operatorname{OGMC}(1,3)$ and $\operatorname{OGMC}_{\mathrm{G}}(1,3)$ models that have new optimized background values based on Simpson's rule are able to produce much more accurate predictions in terms of both MAPE and RMSE values. Thus, Simpson's rule can work as an efficient alternative technique for calculating background values, which has good capability to enhance prediction accuracy. Moreover, the $\mathrm{OGMC}_{\mathrm{G}}(1,3)$ model achieves the highest predicting accuracy with 11.67\%MAPE values and 8019.37RMSE values. Therefore, the e-waste amount in Washington State from 2016 to 2020 is predicted by the superior model, namely, $\mathrm{OGMC}_{\mathrm{G}}(1,3)$.
3.3.3. Future Projections of e-Waste via $\operatorname{OGMC}_{G}(1,3)$. The future e-waste estimations can only be forecasted under the premise of known future values of the population density and median household income. For the future values of the population density, the Washington State Office of Financial Management has provided the trends on the website [38], which is given in Table 10. For the median household income in Table 10, its observations from 2016 to 2017 are available from the above website [36], and its future estimations from 2018 to 2020 are predicted by using the $\operatorname{GM}(1,1)$ model [39], which is a reliable and practical technique for univariate predictions.

Initially, data sets of the median household income from 2003 to 2017 are used for model calibration. Then we will obtain the parameters $[a, b]=[-0.02078,48518.01491]$, and the corresponding time response function is given below: 
TABLe 10: Estimated e-waste, population density, and median household income in case three from 2016 to 2020.

\begin{tabular}{lccc}
\hline$i$ (year) & $\begin{array}{c}\text { Estimated E-waste } \\
X_{1}^{(0)}(\text { tons })\end{array}$ & $\begin{array}{c}\text { Population density } \\
X_{2}^{(0)} \text { (people/se mi) }\end{array}$ & $\begin{array}{c}\text { Median household } \\
\text { Income } X_{3}^{(0)}(\$)\end{array}$ \\
\hline 2016 & 80377.91 & 108.10 & 65500.29 \\
2017 & 85503.45 & 110.00 & 68550.43 \\
2018 & 88941.10 & 111.77 & 66894.16 \\
2019 & 90493.07 & 113.46 & 68298.47 \\
2020 & 92405.41 & 115.11 & 69732.25 \\
\hline
\end{tabular}

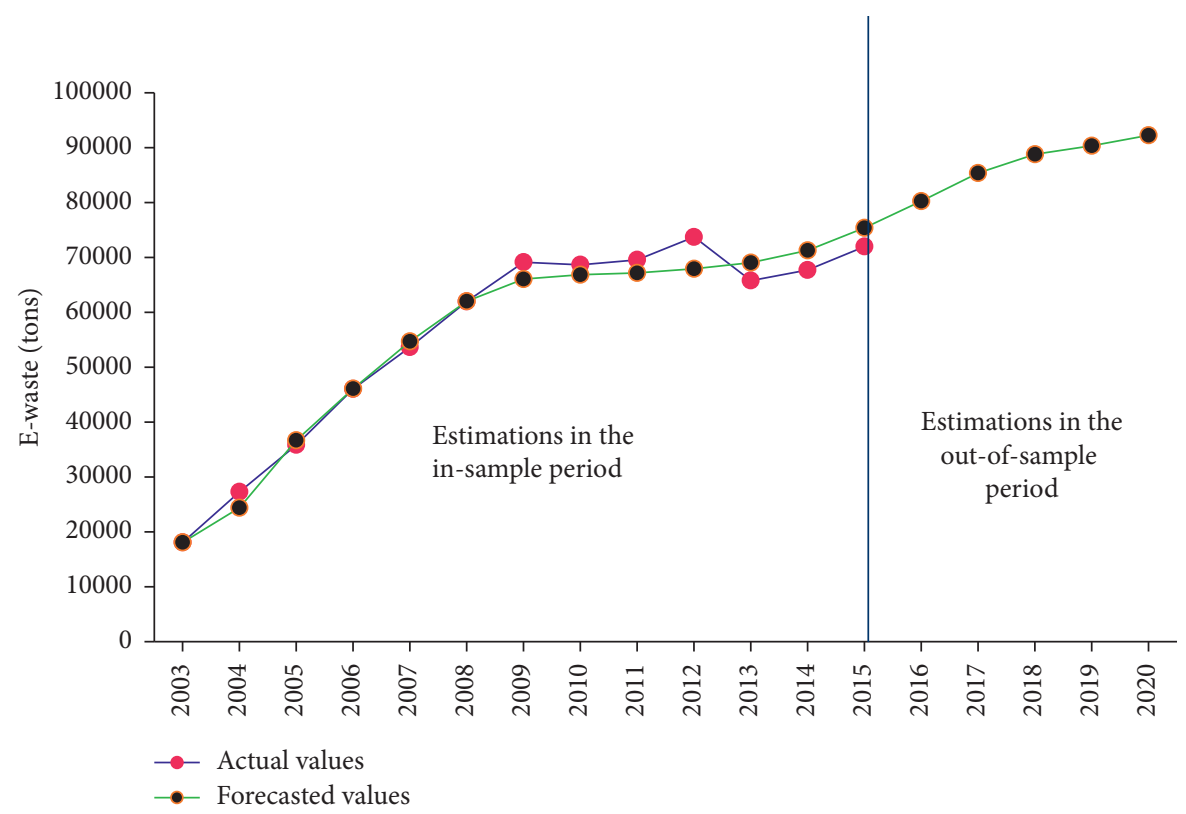

FIGURE 2: Visual comparison of the real observations and predicted values from 2003 to 2020.

$$
\widehat{X}_{1}^{(1)}(t+1)=2.3823 \times 10^{6} \times e^{0.02078 t}-2.3353 \times 10^{6} .
$$

Subsequently, estimated values from 2018 to 2020 are given in Table 10. By the way, the MAPE value in the insample period from 2003 to 2017 reaches 3.14\%, inferring excellent forecasting capability of the $\operatorname{GM}(1,1)$ model.

Once we have the values of these two relevant factors from 2016 to 2020, the future projections of the e-waste amount can be forecasted by using the $\operatorname{OGMC}_{\mathrm{G}}(1,3)$ model. following equations (1)-(4) and (27)-(36), the forecasts from 2016 to 2020 will be calculated in Table 10.

Furthermore, by using the $\mathrm{OGMC}_{\mathrm{G}}(1,3)$ model, the visual comparison of the real observations and predicted values from 2003 to 2020 are presented in Figure 2. As it can be observed from Figure 2, the predicted values fit the real observations well in the in-sample period, and further projections are likely to remain increasing from 2016 to 2020. Thus, the optimized $\operatorname{OGMC}_{\mathrm{G}}(1,3)$ can act as an appropriate tool for e-waste forecast in this paper.

\section{Conclusions and Discussions}

In summary, accurate future estimations play an essential role for decision-makers in framing and implementing sensible plans and policies. Thus, a grey prediction model with convolution integral, shorted for $\operatorname{GMC}(1, n)$, is introduced alongside with its improved variants $\mathrm{GMC}_{\mathrm{G}}(1, n)$. These two models have been applied to solve various prediction issues by many researchers because of their correct solutions to the whitening function. However, inaccurate methods to calculate the background values may incur large deviations of the parameter estimations, which are essential for accurate future projections. Therefore, mathematical analyses of the gap between the actual and estimated background values are discussed in detail. Moreover, Simpson's rule is introduced to compute the background values. Subsequently, the OGMC $(1, n)$ and $\operatorname{OGMC}_{\mathrm{G}}(1, n)$ models are designed by utilizing the optimized background values. Then, a comparative analysis via utilizing the same data points is run to analyze the performance of the proposed models in the three case studies. Through the discussion and analysis of the experimental studies, several conclusions can be drawn:

(i) The new proposed OGMC $(1, n)$ and $\operatorname{OGMC}_{\mathrm{G}}(1, n)$ models generally outperform the previous $\operatorname{GM}(1, n), \quad \operatorname{DG} M(1, n), \quad \operatorname{GMC}(1, n), \quad$ and $\mathrm{GMC}_{\mathrm{G}}(1, n)$ models because of the optimized background values. Moreover, the $\mathrm{OGMC}_{\mathrm{G}}(1, n)$ 
model is normally superior to $\operatorname{OGMC}(1, n)$ due to the accurate Gaussian solution.

(ii) The optimized background values based on Simpson's rule can significantly enhance the forecasting capability of the previous $\operatorname{GMC}(1, n)$ models. Additionally, the reconstructed background values have merits in the simplified structure, high reliability, and wide applicability, which have been proven in three cases. Most importantly, the new method can improve the accuracy of model calibration avoiding the unsatisfactory errors generated from the discrete function to the continuous one.

(iii) The $\mathrm{OGMC}_{\mathrm{G}}(1, n)$ model rises as a more viable option for future projections of e-waste amount in Washington State owing to its accurate and reliable performances in three cases. Moreover, the future e-waste amount remains increasing for the upcoming years.

A limitation of this paper is caused by the linear structure of the proposed model, which may produce errors when dealing with the nonlinear sequences. Therefore, future work will be carried out by proposing a nonlinear $\operatorname{OGMC}(1, n)$ model with optimized background values based on Simpson's rule.

\section{Nomenclature}

\begin{tabular}{|c|c|}
\hline AGO: & Accumulated generating operation \\
\hline IAGO: & Inverse accumulated generating operation \\
\hline GM $(1, n)$ : & $\begin{array}{l}\text { Grey model having one order and } n \\
\text { variables [8] }\end{array}$ \\
\hline $\operatorname{DGM}(1, n)$ : & $\begin{array}{l}\text { Discrete grey model having one order and } \\
n \text { variables [40] }\end{array}$ \\
\hline $\operatorname{GMC}(1, n)$ : & $\begin{array}{l}\text { Grey model with convolution integral } \\
\text { based on trapezoidal rule [14] }\end{array}$ \\
\hline $\mathrm{GMC}_{\mathrm{G}}(1, n):$ & $\begin{array}{l}\text { Grey model with convolution integral } \\
\text { based on the gaussian rule [31] }\end{array}$ \\
\hline $\operatorname{OGMC}(1, n)$ : & $\begin{array}{l}\text { Optimized grey model with convolution } \\
\text { integral based on the trapezoidal rule }\end{array}$ \\
\hline $\mathrm{OGMC}_{\mathrm{G}}(1, n):$ & $\begin{array}{l}\text { Optimized grey model with convolution } \\
\text { integral based on the gaussian rule. }\end{array}$ \\
\hline
\end{tabular}

\section{Data Availability}

The data utilized to support the findings are available in the above context or can be obtained from the corresponding author upon request.

\section{Conflicts of Interest}

The authors declare that they have no conflicts of interest.

\section{Acknowledgments}

This work was sponsored by the National Natural Science Foundation of China (nos. 71901191, 71971154, 71701024, and 71771119) and Project of the Philosophy and Social Sciences in Hangzhou (M20JC086).

\section{References}

[1] J.-L. Deng, "Control problems of grey system," Systems \& Control Letters, vol. 1, no. 5, pp. 288-294, 1982.

[2] B. Zeng, X. Ma, and J. J. Shi, "Modeling method of the grey GM $(1,1)$ model with interval grey action quantity and its application," Complexity, vol. 2020, Article ID 6514236, 10 pages, 2020.

[3] S. Ding, K. W. Hipel, and Y.-G. Dang, "Forecasting China's electricity consumption using a new grey prediction model," Energy, vol. 149, pp. 314-328, 2018.

[4] S. H. Mao, Q. He, X. P. Xiao, and C. Rao, "Study of the correlation between oil price and exchange rate under the new state of the economy," Scientia Iranica, vol. 26, no. 4, pp. 2472-2483, 2019.

[5] M. Zhou, B. Zeng, and W. H. Zhou, "A hybrid grey prediction model for small oscillation sequence based on information decomposition," Complexity, vol. 2020, Article ID 5071267, 13 pages, 2020.

[6] X. Ma, M. Xie, W. Wu, B. Zeng, Y. Wang, and X. Wu, “The novel fractional discrete multivariate grey system model and its applications," Applied Mathematical Modelling, vol. 70, pp. 402-424, 2019.

[7] X. Zhu, Y. Dang, and S. Ding, "Using a self-adaptive grey fractional weighted model to forecast Jiangsu's electricity consumption in China," Energy, vol. 190, p. 116417, 2020.

[8] T.-L. Tien, "A research on the grey prediction model GM (1, n)," Applied Mathematics and Computation, vol. 218, no. 9, pp. 4903-4916, 2012.

[9] B. Zeng and C. Li, "Improved multi-variable grey forecasting model with a dynamic background-value coefficient and its application," Computers \& Industrial Engineering, vol. 118, pp. 278-290, 2018.

[10] S. Ding, "A novel discrete grey multivariable model and its application in forecasting the output value of China's hightech industries," Computers \& Industrial Engineering, vol. 127, pp. 749-760, 2019.

[11] S. H. Mao, X. P. Xiao, and M. Y. Gao, "Nonlinear fractional order grey model of urban traffic flow short-term prediction," Journal of Grey System, vol. 30, no. 4, pp. 1-17, 2018.

[12] W. Meng, D. Yang, and H. Huang, "Prediction of China's sulfur dioxide emissions by discrete grey model with fractional order generation operators," Complexity, vol. 2018, Article ID 8610679, 13 pages, 2018.

[13] S. H. Mao, M. Y. Gao, and X. P. Xiao, "Fractional order accumulation time-lag GM $(1, N, t)$ model and its application," Systems Engineering-Theory \& Practice, vol. 35, no. 2, pp. 430-436, 2015.

[14] T.-L. Tien, "The indirect measurement of tensile strength of material by the grey prediction model GMC $(1, n), "$ Measurement Science and Technology, vol. 16, no. 6, pp. 1322-1328, 2005.

[15] T.-L. Tien, "The deterministic grey dynamic model with convolution integral DGDMC $(1, n)$," Applied Mathematical Modelling, vol. 33, no. 8, pp. 3498-3510, 2009.

[16] T.-L. Tien, "The indirect measurement of tensile strength for a higher temperature by the new model $\operatorname{IGDMC}(1, n)$," Measurement, vol. 41, no. 6, pp. 662-675, 2008.

[17] T.-L. Tien, "The indirect measurement of tensile strength by the new model FGMC $(1, n)$," Measurement, vol. 44, no. 10, pp. 1884-1897, 2011.

[18] Z.-X. Wang, "Nonlinear grey prediction model with convolution integral $\operatorname{NGMC}(1, n)$ and its application to the forecasting of China's industrial $\mathrm{SO}_{2}$ emissions and its 
application to the forecasting of chinas industrial $\mathrm{SO}_{2}$ emissions," Journal of Applied Mathematics, vol. 2014, Article ID 580161, 9 pages, 2014.

[19] L. Wu and Z. Zhang, "Grey multivariable convolution model with new information priority accumulation," Applied Mathematical Modelling, vol. 62, pp. 595-604, 2018.

[20] X. Ma, Z. B. Liu, Y. Wei et al., "A novel kernel regularized nonlinear GMC $(1, n)$ model and its application," Journal of Grey System, vol. 28, no. 3, pp. 97-109, 2016.

[21] J. L. Deng, "Grey modeling in energy-gathering space," Journal of Grey System, vol. 19, pp. 301-308, 2007.

[22] J. L. Deng, "On multi-energy space: resource space," Journal of Grey System, vol. 21, pp. 1-4, 2009.

[23] Z. X. Wang and P. Hao, "An improved grey multivariable model for predicting industrial energy consumption in China," Applied Mathematical Modelling, vol. 40, no. 11-12, pp. 5745-5758, 2016.

[24] G. M. Duman, E. Kongar, and S. M. Gupta, "Estimation of electronic waste using optimized multivariate grey models," Waste Management, vol. 95, pp. 241-249, 2019.

[25] L. Liu, Q. Wang, J. Wang, and M. Liu, “A rolling grey model optimized by particle swarm optimization in economic prediction," Computational Intelligence, vol. 32, no. 3, pp. 391-419, 2016.

[26] K. Li, L. Liu, J. Zhai, T. M. Khoshgoftaar, and T. Li, "The improved grey model based on particle swarm optimization algorithm for time series prediction," Engineering Applications of Artificial Intelligence, vol. 55, pp. 285-291, 2016.

[27] L.-C. Hsu, "A genetic algorithm based nonlinear grey Bernoulli model for output forecasting in integrated circuit industry," Expert Systems with Applications, vol. 37, no. 6, pp. 4318-4323, 2010.

[28] C.-H. Wang and L.-C. Hsu, "Using genetic algorithms grey theory to forecast high technology industrial output," Applied Mathematics and Computation, vol. 195, no. 1, pp. 256-263, 2008.

[29] H. Zhao and S. Guo, “An optimized grey model for annual power load forecasting," Energy, vol. 107, pp. 272-286, 2016.

[30] F. X. Wang and L. S. Zhang, "Combination gray forecast model based on the ant colony algorithm," Mathematics in Practice and Theory, vol. 39, no. 14, pp. 102-106, 2009, in Chinese.

[31] X. Ma and Z. B. Liu, "Research on the novel recursive discrete multivariate grey prediction model and its applications," Applied Mathematical Modelling, vol. 40, no. 7-8, pp. 48764890, 2016.

[32] X. Ma, Z. Liu, and Y. Wang, "Application of a novel nonlinear multivariate grey Bernoulli model to predict the tourist income of China," Journal of Computational and Applied Mathematics, vol. 347, pp. 84-94, 2019.

[33] L. H. Samuel, Metal Data, Reinhold, West New York, NJ, USA, 1952.

[34] R. Intharathirat, P. Abdul Salam, S. Kumar, and A. Untong, "Forecasting of municipal solid waste quantity in a developing country using multivariate grey models," Waste Management, vol. 39, pp. 3-14, 2015.

[35] S.-S. Chung, K.-Y. Lau, and C. Zhang, "Generation of and control measures for, e-waste in Hong Kong," Waste Management, vol. 31, no. 3, pp. 544-554, 2011.

[36] Washington State Office of Financial Management Median Household Income Estimates, 2018, http://Ofm.wa.gov.

[37] Washington State Office of Financial Management Population Estimates, 2018, http://Ofm.wa.gov.
[38] Washington State Office of Financial Management Population forecast, 2018, http://Ofm.wa.gov.

[39] S. Ding, "A novel self-adapting intelligent grey model for forecasting China's natural-gas demand," Energy, vol. 162, pp. 393-407, 2018.

[40] B. Zeng, H. Duan, and Y. Zhou, "A new multivariable grey prediction model with structure compatibility," Applied Mathematical Modelling, vol. 75, pp. 385-397, 2019. 